\section{ENTORSE DE TORNOZELO}

Autoria: Sociedade Brasileira de Ortopedia e Traumatologia

Participantes: Fábio Lucas Rodrigues, Gilberto Waisberg

Descrição da metodologia de coleta de evidência: Realizada pesquisa no MEDLINE, da biblioteca nacional de medicina dos Estados Unidos (U.S. National Library of Medicine), através da base de dados MeSH (Medical Subject Heading Terms). Utilizados os termos: lateral ligament, ankle.

\section{Graus de recomendação e força de evidência:}

A: Estudos experimentais ou observacionais de melhor consistência.

B: Estudos experimentais ou observacionais de menor consistência.

C: Relatos de casos (estudos não controlados).

D: Opinião desprovida de avaliação crítica, baseada em consensos, estudos fisiológicos ou modelos animais.

\section{Introdução}

A entorse é um movimento violento, com estiramento ou ruptura de ligamentos de uma articulação. A entorse de tornozelo é uma das lesões musculoesqueléticas frequentemente encontradas na população ativa, que geralmente envolve lesão dos ligamentos laterais. Ocorre com maior frequência nos atletas de futebol, basquete e vôlei, correspondendo a cerca de 10\% a $15 \%$ de todas as lesões do esporte ${ }^{1}(\mathbf{D})$. No Reino Unido, ela acontece em uma a cada 10.000 pessoas da população geral, isto é, cerca de 5.000 lesões por $\operatorname{dia}^{2}$ (D). A entorse do tornozelo pode evoluir com complicações, com vários graus de limitação funcional.

A estabilidade lateral do tornozelo é dada pelo mecanismo contensor dos ligamentos talo-fibular anterior, posterior e talocalcâneo, associada ao terço distal da fíbula. O mecanismo de lesão habitual é a inversão do pé com flexão plantar do tornozelo, numa intensidade além do normal, que acontece geralmente ao pisar em terreno irregular ou degrau. Este movimento anômalo proporciona uma lesão que se inicia no ligamento talo-fibular anterior e pode progredir para uma lesão do ligamento calcâneofibular, com o aumento da energia do trauma. A lesão do ligamento talofibular posterior é rara, ocorrendo apenas na luxação franca do tornozelo ${ }^{3}(\mathbf{A})$.

\section{Como classificar a entorse de tornozelo?}

A classificação de entorse de tornozelo é baseada no exame clínico da área afetada e divide a lesão em três tipos: grau 1estiramento ligamentar; grau 2-lesão ligamentar parcial e grau 3-lesão ligamentar total ${ }^{4}(\mathbf{C})$.

O quadro clínico encontrado é de dor, com edema localizado na face ântero-lateral do tornozelo, equimose mais evidente após 48 horas e dificuldade para deambular. Quanto mais grave a lesão, mais evidentes ficam os sinais. A associação destes sintomas com o teste da gaveta anterior positivo permite caracterizar uma lesão grau 3 em 96\% dos $\operatorname{casos}^{5}$ (D).

\section{São necessários exames complementares?}

A necessidade de exames complementares para entorse de tornozelo baseia-se na suspeita de fraturas associadas. Das radiografias realizadas em doentes com lesão de tornozelo, 85\% são normais ${ }^{6}(\mathbf{B})$. Com intuito de evitar radiografias desnecessárias, foram criadas regras (regras de Ottawa para tornozelo) que indicam a realização de radiografias apenas quando houver dor em pontos ósseos específicos ou na impossibilidade do apoio de marcha (pelo menos quatro passos). Esta regra mostrou sensibilidade de $99,7 \%$, porém com especificidade variável (10\% a $70 \%)^{7}(\mathbf{A})$.

A ressonância magnética pode ser indicada nos casos de persistência da dor após três meses da lesão inicial, com o objetivo de investigar lesões associadas, como osteocondral, do impacto ântero-lateral e identificar lesões ligamentares crônicas $^{8}(\mathbf{B})$.

\section{Quais são as opções de tratamento da entorse de tornozelo na fase aguda?}

O objetivo do tratamento da lesão ligamentar do tornozelo é o retorno às atividades diárias (esporte/trabalho), com remissão da dor, inchaço e inexistência de instabilidade articular.

O tratamento inicial para todas as lesões consiste em repouso por três dias, aplicação local de gelo, elevação do membro afetado e proteção articular com imobilizador ou tala gessada. O uso de antiinflamatórios não-hormonais mostrou diminuição da dor e edema, com melhora precoce da função $\operatorname{articular}^{9}(\mathbf{A})$.

Nas lesões leves, o tratamento é sintomático, com manutenção da imobilização até a melhora dos sintomas, que dura entre uma e duas semanas. Já nas lesões completas, a proteção articular com imobilizadores semi-rígidos possibilitou retorno mais rápido às atividades físicas e laborativas quando comparada à imobilização gessada, porém a ocorrência de edema, dor e instabilidade em longo prazo foi semelhante nos dois $\operatorname{grupos}^{3}(\mathbf{A})$. Outros tipos de imobilização funcional, como enfaixamento e imobilizadores elásticos, tiveram resultados inferiores aos imobilizadores rígidos e semi-rígidos.

O tratamento cirúrgico comparado ao tratamento conservador não mostrou superioridade no retorno precoce à atividade física, apenas parece evoluir com menor instabilidade residual. 0 tratamento deve ser feito de forma individualizada, avaliando-se cuidadosamente os riscos, que são maiores no tratamento cirúrgico. Portanto, a preferência é dada ao tratamento conservador para as lesões agudas, com atenção a pacientes que possam permanecer sintomáticos ${ }^{3}(\mathbf{A})$.

\section{Quais são as possíveis complicações das entorses?}

Alguns pacientes permanecem com dor ou instabilidade após seis meses do tratamento da lesão ligamentar aguda. As possíveis lesões associadas geralmente são por ordem decrescente de frequência: instabilidade crônica, lesão osteocondral, impacto com processo inflamatório tíbio-fibular distal e impacto anterior com exostose. A investigação diagnóstica destes pacientes pode ser realizada pelo exame clínico associado a métodos diagnósticos, como as radiografias simples e com estresse, ressonância magnética e artroscopia, sendo este último o de maior sensibilidade e especificidade ${ }^{10}(\mathbf{A})$. 
Outro fator que piora o prognóstico das lesões ligamentares do tornozelo é a associação de varo no retropé, que foi determinante na evolução para artrose em longo prazo $(30 \text { anos })^{11}(\mathbf{C})$. A pesquisa por tomografia computadorizada mostrou-se confiável para quantificar o varo. Este fato encoraja a realização da osteotomia valgizante do calcâneo nas instabilidades crônicas associadas a varo do retropé ${ }^{12}(\mathbf{B})$.

\section{Qual é a conduta a ser adotada nas instabilidades crônicas?}

Cerca de $20 \%$ das entorses de tornozelo podem evoluir com algum tipo de instabilidade após seis meses da lesão inicial, acompanhada ou não de frouxidão ligamentar. Os pacientes com boa contensão mecânica - chamada instabilidade funcional têm como causa a falha na propriocepção, e são tratados com métodos fisioterápicos. Mesmo aqueles pacientes com frouxidão ligamentar possuem algum déficit de propriocepção, portanto também devem inicialmente ser submetidos à reabilitação. Os pacientes com instabilidade sintomática persistente podem ser submetidos à correção cirúrgica. Não existe evidência na literatura para determinar qual técnica de tratamento cirúrgico leva a melhores resultados, porém é demonstrado que pacientes submetidos à recuperação funcional com imobilizadores semi-rígidos no pós-operatório tiveram retorno mais precoce às atividades diárias, quando comparados àqueles que utilizaram imobilização $\operatorname{gessada}^{13}(\mathbf{A})$.

\section{Qual é a conduta a ser adotada nas lesões osteocondrais do talus?}

Em pacientes sintomáticos com lesão condral do talus graus 2b, 3 e 4 de Ferkel, pode ser indicada cirurgia. Existem várias técnicas, mas as mais utilizadas são a condroplastia, microfratura ou transferência autóloga osteocondral, que quando comparadas não apresentam diferença nos resultados obtidos após dois anos de acompanhamento ${ }^{14}(\mathbf{A})$.

\section{Existe prevenção eficaz para a entorse?}

Os imobilizadores semi-rígidos podem reduzir em até $47 \%$ a incidência de entorse de tornozelo em atletas praticantes de modalidades esportivas de alto risco. Este valor é ainda maior naqueles que já tiveram uma lesão ligamentar $\operatorname{prévia}^{15}(\mathbf{A})$.
Referências

1. MacAuley D. Ankle injuries: same joint, different sports. Med Sci Sports Exerc 1999;31:S409-11.

2. Kannus P, Renström P. Treatment for acute tears of the lateral ligaments of the ankle. Operation, cast, or early controlled mobilization. J Bone Joint Surg Am 1991;73:305-12.

3. Kerkhoffs GM, Handoll HH, de Bie R, Rowe BH, Struijs PA. Surgical versus conservative treatment for acute injuries of the lateral ligament complex of the ankle in adults. Cochrane Database Syst Rev 2007;(2):CD000380.

4. Bernett P, Schirmann A. Acute sporting injuries of the ankle joint. Unfallheilkunde 1979;82:155-60.

5. Van Dijk CN. How evidence based is our clinical examination of the ankle? In: MacAuley D, Best TM, eds. Evidence-based sports medicine. 14th ed. London, U.K.: BMJ; 2002:445-7.

6. Stiell IG, McKnight RD, Greenberg GH, McDowell I, Nair RC, Wells GA, et al. Implementation of the Ottawa ankle rules. JAMA 1994;271:827-32.

7. Bachmann LM, Kolb E, Koller MT, Steurer J, ter Riet G. Accuracy of Ottawa ankle rules to exclude fractures of the ankle and mid-foot: systematic review. BMJ 2003; 326:(7386)417.

8. Kreitner KF, Ferber A, Grebe P, Runkel M, Berger S, Thelen M. Injuries of the lateral collateral ligaments of the ankle: assessment with MR imaging. Eur Radiol 1999;9:519-24.

9. Slatyer MA, Hensley MJ, Lopert R. A randomized controlled trial of piroxicam in the management of acute ankle sprain in Australian Regular Army recruits. The Kapooka Ankle Sprain Study. Am J Sports Med 1997;25:544-53.

10. Takao M, Uchio Y, Naito K, Fukazawa I, Ochi M. Arthroscopic assessment for intra-articular disorders in residual ankle disability after sprain. Am J Sports Med 2005;33:686-92.

11. Valderrabano V, Hintermann B, Horisberger M, Fung TS. Ligamentous posttraumatic ankle osteoarthritis. Am J Sports Med 2006;34:612-20.

12. Van Bergeyk AB, Younger A, Carson B. CT analysis of hindfoot alignment in chronic lateral ankle instability. Foot Ankle Int 2002;23:37-42. 13. de Vries JS, Krips R, Sierevelt IN, Blankevoort L. Interventions for treating chronic ankle instability. Cochrane Database Syst Rev 2006;(4):CD004124.

14. Gobbi A, Francisco RA, Lubowitz JH, Allegra F, Canata G. Osteochondral lesions of the talus: randomized controlled trial comparing chondroplasty, microfracture, and osteochondral autograft transplantation. Arthroscopy 2006; 22:1085-92.

15. Handoll HH, Rowe BH, Quinn KM, de Bie R. Interventions for preventing ankle ligament injuries. Cochrane Database Syst Rev 2001;(3):CD000018. 